\title{
CRITÉRIOS E ESTRATÉGIAS DE QUALIDADE E RIGOR NA PESQUISA QUALITATIVA
}

\author{
CRITERIA AND STRATEGIES OF QUALITY AND RIGOR IN \\ QUALITATIVE RESEARCH
}

\section{CRITERIOS Y ESTRATEGIAS DE CALIDAD Y RIGOR EN LA INVESTIGACIÓN CUALITATIVA}

\author{
Isabela Silva Cancio Velloso* \\ Janaína Soares Tizzoni**
}

\begin{abstract}
RESUMO
O objetivo deste artigo é discutir estratégias que auxiliem na operacionalizaçáo de critérios que assegurem a qualidade e o rigor na pesquisa qualitativa. Trata-se de uma reflexão teórica acerca dos critérios de credibilidade, transferibilidade, dependibilidade e confirmabilidade propostos como um dos meios de se assegurar o rigor na pesquisa qualitativa. Esses critérios foram propostos a partir de uma analogia com critérios historicamente utilizados na pesquisa qualitativa, considerando-se as especificidades da investigação naturalista. Devem ser considerados em todo o processo da pesquisa, desde sua concepçáo até a interpretaçáo dos dados. No entanto, é inegável o quão desafiadora é a consolidação da utilização desses critérios, pois implica em profundo e intenso envolvimento do pesquisador com os dados produzidos, em um continuum de processos que evocam criatividade, flexibilidade, capacidade crítico-reflexiva, além dos princípios éticos.
\end{abstract}

Palavras-chave: Pesquisa Qualitativa; Metodologia; Conhecimento; Ciências Sociais; Confiabilidade dos dados.

\begin{abstract}
The aim of this paper is to discuss strategies to support the operationalization of criteria that ensure quality and rigor in qualitative research. It is a theoretical reflection on the criteria of credibility, transferability, dependability and confirmability proposed as one of the ways to ensure rigor in qualitative research. These criteria were proposed based on an analogy with criteria historically used in qualitative research, considering the specificities of naturalistic research. They must be considered throughout the research process, from its conception to data analysis. However, it is undeniable how challenging it is to consolidate the use of these
\end{abstract}

*Enfermeira. Doutora. Escola de Enfermagem, Universidade Federal de Minas Gerais, Belo Horizonte, Minas Gerais. Brasil. ORCID: 0000-0001-5408-0825 Email: isavelloso@ufmg.br Autora correspondente.

**Enfermeira. Mestra. Centro Universitário UniBH, Belo Horizonte, Minas Gerais, Brasil. ORCID: 0000-0002-1977-4132 Email: drajst@hotmail.com 
criteria, as it implies a deep and intense involvement of the researcher with produced data, in a continuum of processes that evoke creativity, flexibility, critical-reflexive capacity, in addition to ethical principles.

Key words: Qualitative Research; Methodology; Knowledge; Social Sciences; Data Accuracy.

\section{RESUMEN}

El objetivo de este artículo es discutir estrategias que ayuden en la operacionalización de criterios que garanticen la calidad y el rigor en la investigación cualitativa. Es una reflexión teórica sobre los criterios de credibilidad, transferibilidad, confiabilidad y confirmabilidad propuestos como uno de los medios para garantizar el rigor en la investigación cualitativa. Estos criterios se propusieron en base a una analogía con criterios utilizados históricamente en la investigación cualitativa, considerando las especificidades de la investigación naturalista. Deben considerarse durante todo el proceso de investigación, desde su concepción hasta la interpretación de los datos. Sin embargo, es innegable lo difícil que es consolidar el uso de estos criterios, ya que implica una participación profunda e intensa del investigador con los datos producidos, en un continuo de procesos que evocan creatividad, flexibilidad, capacidad crítico-reflexiva, además de principios éticos.

Palabras clave: Investigación Cualitativa; Metodología; Conocimiento; Ciencias Sociales; Exactitud de los Datos.

Fecha de recepção: 11/05/2020

Fecha de aceitação: 11/07/2020

\section{INTRODUÇÁO}

Amplamente utilizada em investigaçóes na área da saúde -e particularmente na enfermagem- a pesquisa qualitativa como evidência é um tópico complexo e que, ainda hoje, configura-se como alvo de grande controvérsia. Assim, é importante que se compreenda com clareza seus fundamentos, para que se possa melhor analisar sua real dimensão e o potencial de utilização de seus resultados.

Inicialmente, cabe destacar que a forma como os pesquisadores enxergam o conhecimento é influenciado por seus princípios ontológicos relacionados à natureza da realidade, ao que a constitui- e epistemológicos -ligados à natureza do conhecimento acerca da realidade, como o mesmo pode ser construído, adquirido e comunicado $^{(1-3)}$. Naturalmente, os paradigmas da pesquisa qualitativa, quando comparados aos da quantitativa, abarcam diferentes horizontes ontológicos e epistemológicos, com diferentes suposições da realidade e do conhecimento, o que se reflete em suas metodologias e métodos ${ }^{(1)}$. As diferenças ontológicas e epistemológicas entre as abordagens qualitativa e quantitativa contribuem para o debate acerca da pesquisa qualitativa como evidência, bem como trazem para a discussão a diversidade no potencial de utilização de seus resultados.

$\mathrm{Na}$ pesquisa quantitativa, a ênfase está na mensuraçáo e quantificação dos fenômenos ${ }^{(4,5)}$. Os pesquisadores que adotam essa abordagem têm uma visão positivista, na qual se vislumbra a descoberta de um conhecimento absoluto acerca de uma realidade objetiva ${ }^{(1)}$. Por outro lado, fortemente ancorada nas ciências sociais, a pesquisa qualitativa pode ser descrita como interpretativa e naturalista, partindo da premissa de que as açóes humanas são influenciadas por construçóes sociais, tais como significados, intençóes, motivos, crenças, regras e valores sociais e não por leis universais, o que significa dizer que são contexto dependentes ${ }^{(4)}$. A abordagem naturalista, na qual se insere a pesquisa qualitativa, tem como objetivo o desenvolvimento de um conhecimento ideográfico, considerando as especificidades e individualidades dos objetos estudados, com especial atenção às diferenças e similaridades entre eles ${ }^{(6,7)}$.

Salvaguardadas as particularidades de cada uma dessas abordagens, cabe salientar que a produção científica de qualidade deve ser premissa de ambas, uma vez que seus resultados devem ser convertidos 
em conhecimentos a serem utilizados no contexto de práticas profissionais ${ }^{(8)}$. Nesse sentido, considerase que a qualidade da pesquisa perpassa o rigor $^{(9)}$, bem como os critérios que subsidiam esse rigor são importantes para garantir orientação, tanto para pesquisadores, quanto para leitores dos estudos produzidos ${ }^{(10)}$.

$\mathrm{Na}$ ciência da enfermagem, a abordagem naturalista se propóe a explorar fenômenos muito complexos e a produzir entendimentos diferenciados que podem influenciar diretamente a política e a assistência à saúde ${ }^{(11)}$. Com a crescente demanda de formuladores de políticas por resultados de pesquisas que orientem a tomada de decisóes em ambientes complexos da assistência à saúde, critérios que garantam a qualidade da pesquisa qualitativa na enfermagem, o que abarca os critérios de rigor, vêm recebendo maior consideração nos últimos $\operatorname{anos}^{(9)}$.

Embora, questóes acerca da utilização dos resultados da pesquisa qualitativa ainda suscitem discussões, ressalta-se que a utilização de critérios de rigor é um dos caminhos para o aprimoramento de sua qualidade e aplicabilidade. Entretanto, é preciso compreender que a utilização de critérios de rigor em pesquisa se torna mais desafiadora quando se está diante de narrativas do que quando se lida com números e estatísticas ${ }^{(12,13)}$. Se por um lado, nas pesquisas quantitativas, o pesquisador está inserido em uma perspectiva altamente objetiva, na qual trabalha estritamente com dados numéricos e análises estatísticas, na pesquisa qualitativa os dados não-numéricos e a interpretação fenomenológica, ligada de forma inextricável aos sentidos e à subjetividade humana, têm alimentado controvérsias em relação aos critérios de qualidade dos resultados da pesquisa qualitativa ${ }^{(2,5)}$.

Nas últimas décadas, observou-se o empenho de diversos pesquisadores no âmbito de propor orientaçóes para o julgamento do rigor na pesquisa naturalista ${ }^{(14)}$. Dentre esses esforços, na década de 1980, Guba ${ }^{(6)}$, Lincoln e Guba ${ }^{(15)}$ e Guba e Lincoln ${ }^{(16)}$, estabelecendo uma certa analogia aos critérios de validade interna e externa, confiabilidade e objetividade, utilizados na pesquisa quantitativa, propuseram os critérios de credibilidade, transferibilidade, dependibilidade e confirmabilidade como critérios para a pesquisa naturalista.

Concomitante a esse movimento, também se observou crescente demanda por transparência no relato das pesquisas, o que fomentou a criação de guias estipulando critérios para o relato completo e claro dos estudos. Nesse sentido, é importante considerar que a aderência a diretrizes para o relato de pesquisas qualitativas tem potencial para influenciar o padrão das publicaçôes em enfermagem, fortalecendo, em última análise, a posição geral da qualidade dessas pesquisas na área ${ }^{(11)}$.

A utilização de guias com diretrizes e checklists para garantir a qualidade do relato dos resultados de pesquisas qualitativas incluem, entre seus propósitos, auxiliar os leitores na avaliação do rigor metodológico do estudo relatado ${ }^{(17)}$. Dentre os exemplos desse tipo de guia, pode-se citar o RATS, acrônimo para as palavras em inglês relevância, adequação, transparência e solidez, que representam os critérios do checklist. Outro, o Standards for Reporting Qualitative Research ( $S R Q R)$, dispõe de 21 itens considerados essenciais para o relato completo e transparente da pesquisa qualitativa. O Consolidated Criteria for Reporting Qualitative Research (COREQ), com uma lista de verificação de 32 itens, foi publicado pela rede EQUATOR Network, uma organização que busca melhorar a confiabilidade e a validade da pesquisa biomédica, incluindo a ciência da enfermagem. Desses três exemplos, embora não haja unanimidade, as orientaçôes do COREQ são as de maior grau de consenso entre os pesquisadores qualitativos ${ }^{(11)}$.

Assim, reconhecendo o potencial dos critérios de credibilidade, transferibilidade, dependibilidade e confirmabilidade para ampliar a qualidade e o rigor na pesquisa qualitativa, o que se propóe neste artigo é discutir as estratégias propostas para a operacionalização de cada um deles, favorecendo assim, uma análise criteriosa por parte do pesquisador naturalista, acerca de sua aplicabilidade nas pesquisas desenvolvidas.

\section{CRITÉRIOS DE QUALIDADE E RIGOR NA PESQUISA QUALITATIVA}

Ao proporem os quatro critérios de análise de rigor da pesquisa qualitativa, Guba ${ }^{(6)}$, Lincoln e Guba ${ }^{(15)}$ e Guba e Lincoln ${ }^{(16)}$ estabeleceram uma relação entre tais critérios e aqueles utilizados na pesquisa quantitativa, bem como definiram um aspecto central na análise de cada um deles (Quadro 1). 
Quadro 1. Relaçáo entre critérios de rigor naturalistas e positivistas.

\begin{tabular}{lcc}
\hline Critério naturalista & Aspecto central & Critério positivista \\
\hline Credibilidade & Valor da verdade & Validade interna \\
Transferibilidade & Aplicabilidade & Validade externa \\
Dependibilidade & Consistência & Confiabilidade \\
Confirmabilidade & Neutralidade & Objetividade \\
\hline
\end{tabular}

Ao estabelecer a analogia com os critérios de rigor da pesquisa quantitativa, não se pretende apenas criar um vocabulário específico para a pesquisa qualitativa, mas sim ressignificá-los, considerando as especificidades da investigação naturalista.

Credibilidade: A credibilidade, relacionada à validade interna da pesquisa, refere-se à confiança que pode ser depositada na veracidade de seus resultados ${ }^{(18)}$, sendo este um atributo necessário a investigaçóes de qualquer natureza. A discussão acerca da validade de uma pesquisa está relacionada à precisão e veracidade de seus achados científicos, os quais devem expressar a verdade acerca da realidade estudada $^{(19,20)}$. Nesse sentido, a credibilidade dos achados de pesquisa também se prende aos procedimentos metodológicos empregados, o que pode ser um desafio na pesquisa qualitativa, uma vez que reivindica a associaçáo do rigor inerente ao processo científico à subjetividade e à criatividade, que são intrínsecas a esta abordagem, com possíveis adaptaçóes dos métodos utilizados em acordo com as particularidades de cada contexto ${ }^{(8,13,19)}$.

No que se refere ao aspecto central da credibilidade, qual seja o valor da verdade, a questão principal está em torno de como é possível estabelecer a confiança, a credibilidade na verdade posta nos achados de uma investigação particular, com os sujeitos e o contexto a partir dos quais a mesma foi realizada ${ }^{(6)}$. Com o valor da verdade, o pesquisador, mais do que manter o foco na acurácia dos resultados e sua relação com os dados, reconhece a existência de múltiplas realidades e busca a credibilidade desses achados nas próprias fontes das quais os dados foram extraídos, apresentando, de forma clara e precisa, as perspectivas dos participantes $^{(6,8,15,21)}$.

Transferibilidade: Análoga à validade externa da pesquisa positivista, a transferibilidade está relacionada à generalizaçáo dos resultados da pesquisa, ou seja, refere-se à possibilidade de transferência dos resultados de uma pesquisa qualitativa para outros contextos, com outros participantes $^{(22)}$. A transferibilidade é pautada no princípio da aplicabilidade, ou seja, no fato de que os achados podem ser aplicadas a outros contextos, configuraçôes ou grupos ${ }^{(8)}$. Nessa perspectiva, cabe ao pesquisador fornecer informaçóes detalhadas sobre o contex to no qual a pesquisa foi desenvolvida para que o leitor possa analisar seu potencial de transferibilidade ${ }^{(6,14,15)}$.

No entanto, é importante considerar que, na perspectiva naturalista, não se tem a intenção de criar generalizaçóes que se sustentem em todos os tempos e lugares, mas sim de formar hipóteses de trabalho que possam ser transferidas de um contexto para outro, dependendo do grau de adequaçáo, ou seja, de similaridade entre esses contextos ${ }^{(6,15}$, 16). Assim, é importante considerar que, diante do conhecimento ideográfico produzido a partir das pesquisas qualitativas, a generalização se dá muito mais no nível do caso do que de populações, de modo que a náo generalização dos achados de uma investigação para outros grupos não deve ser considerada como uma limitação do estudo ${ }^{(7)}$.

Dependibilidade: $O$ terceiro critério de rigor a ser considerado na pesquisa qualitativa é o da dependibilidade, que também é conhecido como auditabilidade e está diretamente relacionado à consistência da pesquisa ${ }^{(23)}$. Embora haja, na pesquisa naturalista, preocupação com a estabilidade dos dados, é preciso levar em conta certas instabilidades aparentes, devido às diferentes realidades que estão sendo exploradas ou a mudanças instrumentais decorrentes do desenvolvimento de insights por parte do investidor como instrumento ${ }^{(6)}$.

Analogamente à confiança, a dependibilidade refere-se à consistência de se observar o mesmo resultado sob circunstâncias similares ${ }^{(15)}$. A dependibilidade abrange, em seu conceito, tanto elementos da estabilidade implícita no termo 
racionalista confiável, quanto da rastreabilidade exigida por mudanças explicáveis na instrumentação ao longo da pesquisa ${ }^{(6)}$.

Confirmabilidade: Por fim, o último critério a ser considerado é o da confirmabilidade, que se refere à possibilidade de os resultados da pesquisa poderem ser confirmados ou corroborados por outros $^{(20)}$, podendo seu conceito ser comparado ao de objetividade utilizado na pesquisa positivista ${ }^{(15)}$. Diante da reconhecida impossibilidade de se alcançar níveis de objetividade semelhantes àqueles almejados na pesquisa quantitativa, o foco da investigação qualitativa se desloca da neutralidade do pesquisador para a confirmabilidade dos dados e das interpretaçóes por ele apresentadas ${ }^{(14)}$.

Tradicionalmente, a confirmabilidade se relaciona à ausência de viés ou de suposiçóes do pesquisador ${ }^{(23)}$. $\mathrm{Na}$ investigação qualitativa, isso pode ser minimizado se o pesquisador proceder à verificação gradual ao longo da coleta de dados, de modo que os dados possam ser corrigidos durante os processos de coleta e análise. Nesse processo, caberá ao pesquisador estar fortemente vigilante em relação a comparaçóes e conclusóes, mantendo uma perspectiva indutiva e verificando todos os dados durante os processos de coleta de dados ${ }^{(24,25)}$.

\section{ESTRATÉGIAS PARA A OPERACIONALI- ZAÇÃO DO RIGOR NA PESQUISA}

Para que se garanta a aplicação dos critérios de rigor em uma investigação qualitativa, é possível associar a eles determinadas estratégias que viabilizem sua operacionalizaçãoo $^{(6,15,16)}$, as quais são apresentadas no Quadro 2.

O uso dessas estratégias é recomendado com o intuito orientar o processo de investigaçáo qualitativa desde sua concepção até a interpretação dos dados, o que, certamente, trará implicaçóes para a pesquisa e o pesquisador, requerendo sua capacidade de resposta sempre que forem necessárias intervençóes que garantam o rigor na pesquisa. A seguir, serão apresentados os principais elementos de cada uma das estratégias propostas.

\section{Envolvimento prolongado no campo e} observaçáo persistente: No que se refere ao envolvimento prolongado no campo, cabe considerar que determinados processos como o conhecimento da cultura, a apropriação das lacunas nas informaçóes provenientes tanto de distorçôes do pesquisador quanto dos informantes e o estabelecimento da relaçáo de confiança com os participantes requerem um investimento de tempo suficiente para que tais propósitos sejam alcançados $^{(2,15)}$.

A permanência no campo por um período prolongado, por um lado, permite que os participantes da pesquisa se adaptem à presença do pesquisador e não o percebam como uma ameaça; por outro, ajuda o pesquisador a entender os principais problemas que podem afetar a qualidade dos dados ${ }^{(6,22)}$. No entanto, é importante salientar que não é possível estipular um período de tempo

Quadro 2. Critérios y estratégias de operacionalização associadas aos critérios de rigor.

\begin{tabular}{|c|c|c|}
\hline Critério & Estratégias propostas & \\
\hline \multirow[t]{6}{*}{ Credibilidade } & Envolvimento prolongado no campo & \multirow{12}{*}{ Diário reflexivo } \\
\hline & Observação persistente & \\
\hline & Triangulação & \\
\hline & Análise por pares & \\
\hline & Análise de caso negativo & \\
\hline & Verificação dos membros & \\
\hline Transferibilidade & Descrição densa & \\
\hline \multirow{3}{*}{ Dependibilidade } & Sobreposição de métodos & \\
\hline & Replicação gradativa & \\
\hline & Auditoria de trilha & \\
\hline \multirow[t]{2}{*}{ Confirmabilidade } & Triangulação & \\
\hline & Auditoria da trilha & \\
\hline
\end{tabular}


ideal para o trabalho de campo, uma vez que essa delimitação está relacionada ao escopo e à sofisticação do contexto ${ }^{(15,22)}$.

Se, assim, o envolvimento prolongado permite alcançar a amplitude do campo, a observaçáo persistente acrescenta a dimensão da relevância ao que, de outra forma, poderia parecer pouco mais do que uma imersão irracional, ou seja, possibilita a compreensão em profundidade acerca do fenômeno estudado $^{(15)}$. É por meio da observação persistente que se potencializa a possibilidade de o investigador descobrir qualidades e características incomuns dos participantes. Qualidades essas associadas ao envolvimento prolongado com o contexto e com os participantes: algo, de resto, vantajoso para a compreensão das características essenciais do ambiente ${ }^{(22)}$.

Triangulação: Outra estratégia que pode agregar credibilidade aos achados e interpretaçóes de pesquisa é a triangulaçáo que, no caso da pesquisa naturalista, envolve principalmente a utilização de múltiplos e diferentes métodos, fontes e investigadores $^{(2,15,22,24)}$. A triangulação ajuda $\mathrm{o}$ investigador a reduzir o viés, bem como possibilita a análise cruzada da integridade das respostas dos participantes ${ }^{(22)}$. No caso das pesquisas qualitativas, a triangulação não vislumbra a busca de uma realidade única ou de um fato objetivo, mas pretende enriquecer os dados ao adicionar características que transformam a simples descrição em um dado saturado ${ }^{(7)}$.

A triangulação de métodos abrange, na medida do possível, o uso de diferentes estratégias de coleta de dados, tais como entrevistas, questionários, análise documental, grupos focais, etc. A triangulação de fontes se refere ao uso de diferentes fontes de evidência para aprimorar a qualidade do dado e para que possíveis predileçóes do investigador sejam testadas da forma mais árdua possível. Por fim, o uso de diferentes pesquisadores para coletar, analisar e interpretar o dado de uma investigação traz diferentes perspectivas sobre o problema em questão ${ }^{(6,15,20,24)}$. No âmbito da pesquisa qualitativa, recomenda-se que sejam incluídas uma ou duas técnicas de triangulação para se responder uma questão ${ }^{(22)}$.

Revisão por pares: A revisão por pares está ancorada no princípio segundo o qual -ao longo do processo de pesquisa- o pesquisador qualitativo deve, acima de tudo, buscar apoio de outros profissionais -membros de grupos de pesquisa, orientadores, professores, etc.- que possam lhe oferecer apoio acadêmico e feedback que o ajude a melhorar a qualidade de sua investigação ${ }^{(22)}$.

Nesse sentido, a revisão por pares envolve um processo exposiçáo a um pesquisador que não esteja diretamente envolvido na investigação, o qual fará uma análise com o intuito de explorar aspectos da pesquisa que, de outra forma, poderiam permanecer implícitos, se considerada apenas na perspectiva do próprio autor da pesquisa ${ }^{(15)}$. Esse processo permite que os pesquisadores tenham a oportunidade tanto de testarem a evolução de suas ideias em relação à investigaçáo, quanto de se exporem a outras questóes de pesquisa ainda não pensadas ${ }^{(6)}$.

Análise de caso negativo: A análise de caso negativo é uma estratégia analítica crítica para a validade de uma pesquisa naturalista. É a chave para a compreensão dos casos mais comuns que, quando comparados com os casos negativos, revelarão diferenças importantes; e é o desenvolvimento do entendimento dessas diferenças que geralmente é crítico para a compreensão do processo como um $\operatorname{todo}^{(25)}$.

A análise de caso negativo pode ser compreendida como um processo de revisão de hipóteses em retrospectiva, com objetivo de refiná-las continuamente, até que abranjam todos os casos conhecidos. Assim, à medida que a investigação se desenvolve, o pesquisador aperfeiçoa a hipótese de trabalho, considerando evidências negativas que não confirmam os pressupostos originais ${ }^{(2)}$.

Verificaçáo dos membros: A verificação dos membros consiste na apresentação de dados e interpretaçóes aos participantes de um estudo, para que eles possam confirmar a credibilidade das informaçôes e do relato narrativo; o que desloca o processo de validaçáo do pesquisador para o participante ${ }^{(26)}$. É importante compreender que a verificação dos membros deve se constituir enquanto processo contínuo, ao longo da pesquisa, dando a oportunidade aos participantes de corrigirem, em tempo hábil, eventuais equívocos nos dados, análises e interpretaçóes, de forma a garantir a credibilidade dos relatos ${ }^{(2,15)}$. Com a confirmação da precisão dos dados ou resultados pelos participantes, os mesmos podem ser considerados confiáveis e a validade da pesquisa $\operatorname{assegurada}^{(15,27,28)}$.

No dia a dia da pesquisa, vários procedimentos 
podem ser empregados com essa finalidade, sendo uma estratégia comum reunir um grupo de participantes para revisar os resultados, solicitando-lhes que visualizem os dados brutos -transcriçôes, notas de campo observacionais, etc.- e que comentem sua precisão ${ }^{(26)}$.

Descrição densa: A descrição densa é principal estratégia para garantir que os leitores de uma pesquisa julguem a possibilidade de transferibilidade dos achados de um estudo para outros cenários ou contextos semelhantes ${ }^{(2,26)}$. A descrição rica, detalhada e concreta de pessoas e de lugares é um mundo que se abre para o leitor, permitindolhe entender o fenômeno estudado e conformar suas próprias interpretaçóes sobre sentidos e significados ${ }^{(29)}$, de modo que a credibilidade é estabelecida pelas lentes dos interlocutores que leem uma narrativa e são transportados para um cenário ou situação ${ }^{(26)}$. A descrição densa implica a elucidação e detalhamento, por parte do pesquisador, de todas as etapas do processo de pesquisa, desde os procedimentos de coleta de dados, do contexto do estudo, até a produção do relatório final ${ }^{(22)}$.

O processo de escrever usando uma descrição densa e rica tem por finalidade fornecer o maior detalhamento possível, o que pode envolver a descrição de interações, experiências ou ações muito sutis, localização de indivíduos em situaçóes específicas, trazer vividamente um relacionamento ou interação entre pessoas. $\mathrm{O}$ uso desse procedimento evoca uma perspectiva construtivista na contextualização de pessoas ou locais estudados ${ }^{(26)}$.

Sobreposição de métodos: A sobreposição de métodos é uma estratégia empregada na perspectiva $\mathrm{da}$ dependibilidade da pesquisa. $\mathrm{Na}$ verdade, a sobreposição é um tipo de processo de triangulação em que diferentes métodos são usados em conjunto $^{(6,15)}$. No entanto, é importante acentuar que a triangulação é tipicamente realizada para estabelecer credibilidade, e não dependibilidade, ou confiabilidade. Em geral, essa abordagem é recomendada para superar a pouca validade de determinado método, o que pode ser feito com a utilização de dois ou mais métodos agrupados, de tal maneira que a fraqueza de um é compensada pelas potencialidades de outro ${ }^{(6)}$.

Replicação gradativa: Outra estratégia que visa a ampliar tanto a dependibilidade quanto a confirmabilidade da pesquisa qualitativa é a replicação gradativa, técnica na qual a equipe de pesquisa é dividida em dois outros menores e cada uma delas irá lidar, separadamente, com fontes de dados que também foram divididas em duas ${ }^{(6,30)}$. A comunicação entre os dois grupos acontece ao longo do processo de pesquisa, a fim de cruzar as ideias em desenvolvimento e decidir, apropriadamente, as próximas etapas. Tais procedimentos são documentados de forma adequada, não sendo permitido às duas equipes prosseguir com a investigação até o fim antes que os resultados sejam comparados $^{(14)}$.

Auditoria da trilha: A auditoria da trilha é uma estratégia que visa a aumentar o rigor e a transparência na pesquisa qualitativa por meio da descrição de uma trilha na qual se documenta, cronológica e sistematicamente, todos os passos percorridos ao longo da pesquisa, com o intuito de atingir as interpretaçóes apresentadas, permitindo, assim, uma auditoria de todo o processo ${ }^{(31)}$.

Ao estabelecer uma trilha de auditoria, os pesquisadores fornecem documentação clara de todas as decisóes e atividades de pesquisa, de modo a apresentar evidências da trilha ao longo do relatório ou nos apêndices ${ }^{(26)}$. Ademais, cabe considerar que a condução de uma auditoria completa requer que documentos como dados brutos, notas de entrevistas e observaçóes, documentos e registros de campo, notas de testes e outros sejam mantidos, viabilizando, nesse sentido, a verificação cruzada do processo de investigação ${ }^{(6,22)}$.

Diário reflexivo: Como foi possível observar, as estratégias e técnicas discutidas até aqui são aplicadas especificamente a determinados critérios de rigor. No entanto, uma última técnica deve ser ainda considerada: a do diário reflexivo. Tal técnica tem ampla inserção nos quatro critérios mencionados: credibilidade, transferibilidade, dependibilidade e confirmabilidade ${ }^{(15)}$. O diário é um documento reflexivo mantido pelo pesquisador, no qual são feitos registros que possibilitem reflexão, interpretação provisória e planejamento de coleta de dados, de maneira a atestar o que se está aprendendo e o que está acontecendo ao longo do processo de pesquisa ${ }^{(32)}$.

Também fazem parte do processo da análise reflexiva as limitaçóes impostas ao longo do processo no trabalho de campo. Nesse sentido, devem-se considerar os fatores que interferem 
no delineamento do estudo e que implicam em definiçóes metodológicas, além de negociaçóes e decisôes éticas sobre quais dados se obter e de que forma obtê-los; o que deve ser reconhecido pelo pesquisador e registrado no diário reflexivo ${ }^{(7)}$.

Embora náo haja uma estrutura rígida para a construção do diário reflexivo, sugere-se que o mesmo esteja organizado em partes separadas e que inclua: 1) cronograma diário e logístico da pesquisa; 2) anotaçóes que permitam reflexóes sobre os próprios valores e interesses e para a especulação de ideias crescentes; e, por fim, 3) um registro metodológico, com anotação de decisôes e justificativas associadas ${ }^{(15)}$.

\section{CONCLUSÃO}

As questóes apresentadas ao longo desse artigo apontam a importância de que pesquisadores que utilizam a abordagem qualitativa assumam a responsabilidade com os princípios e desenvolvimento da ciência, agindo de forma crítica e proativa para contribuírem com o alcance de níveis satisfatórios de qualidade em seus estudos, o que implica na adoção de critérios e estratégias de rigor. A definição clara dos critérios utilizados ao longo da pesquisa é importante para a própria condução da investigação, mas também permite que os leitores do estudo tenham uma compreensão ampliada de seus achados, além de subsidiar a avaliação dos pares, quando da submissão para publicação em periódicos científicos.

Certamente, as reflexóes ora apresentadas não têm por finalidade encerrar a discussáo acerca do rigor e da qualidade no universo da pesquisa qualitativa, bem como os critérios aqui elencados não representam uma opçáo singular para o seu aprimoramento, embora representem uma

\section{REFERÊNCIAS}

1. Kamal SSLBA. Research paradigm and the philosophical foundations of a qualitative study. Int J Soc Sci [Internet]. 2019 [citado $2019 \mathrm{dez}$ 10]; 4(3): 1386-1394. Disponível em: https:// grdspublishing.org/index.php/people/article/ view/1776

2. Silva A, Castro-Silva CR, Moura L. Pesquisa alternativa viável. Entretanto, não há dúvidas de que a questão do rigor precisa ocupar uma posição de destaque no contexto das práticas de investigação qualitativa, perpassando todo o processo, desde sua concepção mais inicial até a interpretação dos dados, considerando-se que a utilização desses critérios pode representar um salto de qualidade nas pesquisas.

Mesmo reconhecendo a importância e viabilidade de critérios que assegurem o necessário rigor na pesquisa qualitativa, não se pode negar o quão desafiadora é a consolidação de sua aplicação no cotidiano da pesquisa qualitativa, uma vez que implica no profundo e intenso envolvimento do pesquisador com os dados produzidos, em um continuum de processos que evocam sua criatividade, flexibilidade, capacidade críticoreflexiva, além de princípios éticos.

É crescente a produção de pesquisas qualitativas de forma geral, o que se aplica também à enfermagem, cujo conhecimento produzido tem potencial para auxiliar na compreensão de como surgem e se desenvolvem problemas específicos. No entanto, um dos desafios enfrentados na produção científica qualitativa é a condução e divulgação de resultados cuidadosos, cujo rigor permita sua utilização nas práticas profissionais, o que inclui a formulação de políticas de saúde.

Nesse sentido, a importância do emprego de critérios e estratégias de rigor claros no desenvolvimento de pesquisas qualitativas é indiscutível. Assim, sugere-se que as estratégias discutidas neste artigo sejam analisadas criticamente e considerada sua aplicabilidade. Entretanto, não se trata de considerá-las de forma absoluta, pois diante da diversidade e subjetividade que envolve a pesquisa naturalista, a escolha dos caminhos para se chegar ao indispensável rigor metodológico não se pode desconsiderar a lente epistemológica da dessa abordagem.

qualitativa em saúde: percursos e percalços da formação para pesquisadores iniciantes. Saude soc [Internet]. 2018 [citado $2019 \mathrm{dez} 22$ ]; 27(2): 632-645. Disponível em: http://www.scielo. $\mathrm{br} / \mathrm{scielo}$.php?script $=$ sci_arttext $\&$ pid $=$ S0104$12902018000200632 \& \operatorname{lng}=$ en.

3. Berryman DR. Ontology, Epistemology, Methodology, and Methods: Information for Librarian Researchers. Med Ref Serv Quart 
[Internet]. 2019 [citado $2019 \mathrm{dez} 22$ ]; 38:3, 271279. Disponível em: https://doi.org/10.1080/0276 3869.2019.1623614

4. Draper AK. The principles and application of qualitative research. P Nutr Soc [Internet]. 2004 [citado 2019 jun 26]; 63(4): 641-6. Disponível em: https://www.ncbi.nlm.nih.gov/pubmed/15831137

5. Leung L. Validity, reliability, and generalizability in qualitative research. J Family Med Prim Care [Internet]. 2015 [citado 2019 jun 26]; 4(3): 3247. Disponível em: https://www.ncbi.nlm.nih.gov/ pmc/articles/PMC4535087/

6. Guba E. Criteria for assessing the trustworthiness of naturalistic inquiries. ECTJ [Internet]. 1981 [citado 2019 jul 10]; 29(2): 75-92. Disponível em: http://www.jstor.org/stable/30219811

7. Benjumea CC. The quality of qualitative research: from evaluation to attainment. Text Context Nursing [Internet]. 2015 [citado 2019 jul 9]; 24(3): 883-90. Disponível em: http://dx.doi. org/10.1590/0104-070720150001150015

8. Jameel B, Shaheen S, Majid U. Introduction to Qualitative Research for Novice Investigators. URNCST Journal [Internet]. 2018 [citado 2019 dez 20]; 2(6): 1-6. Disponível em: https://www. researchgate.net/publication/326020915

9. Squires A, Dorsen C. Qualitative research in nursing and health professions regulation. Contin Educ [Internet]. 2018 [citado $2019 \mathrm{dez}$ 22]; 9(3): 15-26. Disponível em: https://www. journalofnursingregulation.com/article/S21558256(18)30150-9/fulltext

10. Löblich M. Rigor in qualitative research. In: Matthes J, Editor. The International Encyclopedia of Communication Research Methods. Hoboken: John Wiley \& Sons; 2017. p. 1-9.

11. Smith GD, Gelling L, Haigh C, Barnason S, Allan H, Jackson D. The position of reporting guidelines in qualitative nursing research. J Clin Nur Res [Internet]. 2017 [citado 2020 jul 9]; 27: 889-91. Disponível em: https:// www.sciencedirect.com/science/article/abs/pii/ S0300571219300429?via\%3Dihub

12. Cypress BS. Rigor or reliability and validity in qualitative research: Perspectives, strategies, reconceptualization, and recommendations. DCCN [Internet]. 2017 [citado 2019 jul 1]; 36(4): 253-63. Disponível em: https://www.ncbi.nlm. nih.gov/pubmed/28570380

13. Mackieson P, Shlonsk A, Connolly M. Increasing rigor and reducing bias in qualitative research: a document analysis of parliamentary debates using applied thematic analysis. J Soc Work [Internet]. 2018 [citado $2019 \mathrm{dez}$ 18]; 0(0): 1-16. Disponível em: https://journals.sagepub.com/doi/
abs/10.1177/1473325018786996?journalCode =qswa

14. Moreira H. Critérios e estratégias para garantir o rigor na pesquisa qualitativa. R Bras Ens Ci Tecnol [Internet]. 2018 [citado 2019 mai 29]; 11(1): 40524. Disponível em: https://periodicos.utfpr.edu.br/ rbect/article/view/6977

15. Lincoln YS, Guba EG. Naturalistic inquiry. Beverly Hills, CA: Sage; 1985.

16. Guba E, Lincoln Y. Fourth generation evaluation. Newbury Park, CA: Sage; 1989.

17. Buus N, Perron A. The quality of quality criteria: replicating the development of the Consolidated Criteria for Reporting Qualitative Research (COREQ). Int J Nurs [Internet]. 2019 [citado 2020 jul 9]; 7489(19): 30259-7. Disponível em: https://doi.org/10.1016/j.ijnurstu.2019.103452

18. Patino CM, Ferreira JC. Validade interna e externa: você pode aplicar resultados de pesquisa para seus pacientes? J Bras Pneumol [Internet]. 2018 [citado 2019 jul 10]; 44(3): 183. Disponível em: http://www.scielo.br/scielo.php?pid=S180637132018000300183\&script=sci_arttext\&tlng=pt

19. Korstjens I, Moser A. Series: Practical guidance to qualitative research. Part 4: Trustworthiness and publishing. Eur J Gen Pract [Internet]. 2018 [citado 2019 dez 18]; 24:1, 120-124. Disponível em: https:// doi.org/10.1080/13814788.2017.1375092

20. Forero R, Nahidi S, De Costa J, Mohsin M, Fitzgerald G, Gibson N, et al. Application of fourdimension criteria to assess rigour of qualitative research in emergency medicine. BMC Health Serv Res [Internet]. 2018 [citado 2019 dez 1]; 18(1): 120. Disponível em: https://www.ncbi.nlm. nih.gov/pmc/articles/PMC5816375/

21. D’Abreu IM, Branco LACB, Pacanowski M, Rocha VF, Troccoli IR, Altaf JG. Triangulação: um levantamento do seu uso em artigos publicados nos EnANPADs. Rev Eletr Mach Sobr [Internet]. 2017 [citado 2019 jun 27]; 13(2): 1-12. Disponível em: http://www.machadosobrinho.com.br/revista online/publicacao/artigos/Artigo01REMS13.pdf

22. Anney VN. Ensuring the Quality of the Findings of Qualitative Research: Looking at Trustworthiness Criteria. JETERAPS [Internet]. 2014 [citado 2019 jun 9]; 5(2): 272-81. Disponível em: http://repository.udsm.ac.tz:8080/xmlui/ handle/123456789/256

23. Prion S, Adamson KA. Making sense of methods and measurement: rigor in qualitative research. Clin Simul Nurs [Internet]. 2014 [citado 2019 jul 10]; 10(2): e107-8. Disponível em: https:// www.nursingsimulation.org/article/S18761399(13)00107-2/abstract

24. Denzin N. The research act: a theoretical intro- 
duction to sociological methods. $2^{\text {a }}$ ed. Chicago: Aldine; 1970.368 p.

25. Morse JM. Critical analysis of strategies for determining rigor in qualitative inquiry. Qual Health Res [Internet]. 2015 [citado 2019 jun 26]; 25(9): 1212-22. Disponível em: https://www.ncbi. nlm.nih.gov/pubmed/26184336

26. Creswell JW, Miller DL. Determining validity in qualitative inquiry. Theor Pract [Internet]. 2000 [citado 2019 set 2]; 39(3): 124-30. Disponível em: https://www.tandfonline.com/doi/abs/10.1207/ s15430421tip3903_2

27. Hayashi P, Abib G, Hoppen, N. Validity in qualitative research: a processual approach. The Qualit Rep [Internet]. 2019 [citado 2019 dez 22]; 24(1): 98-112. Disponível em: https://nsuworks. nova.edu/tqr/vol24/iss1/8

28. Smith B, McGannon KR. Developing rigor in qualitative research: problems and opportunities within sport and exercise psychology. Int Ver
Sport Exerc Psychol [Internet]. 2018 [citado 2019 set 2]; 11(1): 101-21. Disponível em: https:// www.tandfonline.com/doi/abs/10.1080/175098 4X.2017.1317357

29. Patton MQ. Qualitative research and evaluation methods. $4^{\mathrm{a}}$ ed. Thousand Oaks, CA: Sage Publications; 2014. 832 p.

30. Ary D, Jacobs L, Sorensen C. Introduction to research in education. $8^{\text {a }}$ ed. Belmont, CA: Cengage Learning; 2010. 669 p.

31. Bowen GA. Supporting a grounded theory with an audit trail: An illustration. Int J Soc Res Methodol [Internet]. 2009 [citado 2019 jul 9]; 12(4): 305-16. Disponível em: https://www.tandfonline.com/doi/ abs/10.1080/13645570802156196

32. Ortlipp M. Keeping and using reflective journals in the qualitative research process. TQR [Internet]. 2008 [citado 2019 set 2]; 13(4): 695705. Disponível em: https://nsuworks.nova.edu/ tqr/vol13/iss $4 / 8$ 